\title{
New aspirations: the debate on aspiration pneumonia treatment guidelines
}

\section{spiration pneumonia is a variant of both} community- and health care-acquired pneumonia that occurs predominantly in older patients and those with a history of swallowing difficulty. ${ }^{1}$ The current Australian guidelines for initial treatment of aspiration pneumonia suggest the use of benzylpenicillin in combination with metronidazole. ${ }^{2}$ Here, we examine the evidence for including metronidazole in empirical treatment of aspiration pneumonia. Although benzylpenicillin has activity against some anaerobes, our use of the term "specific" anti-anaerobic therapy refers to the use of agents with a broad spectrum of activity against anaerobes, such as metronidazole.

\section{What is aspiration pneumonia?}

Several definitions exist for aspiration pneumonia. A commonly accepted definition is infective pneumonia in a patient with a predisposition for aspiration, ${ }^{3-5}$ such as those who have had strokes or who have multiple sclerosis affecting bulbar function. The pneumonia occurs after inhalation of oropharyngeal or gastric secretions colonised by pathogenic bacteria into the larynx and lower respiratory tract. ${ }^{6}$ This should be distinguished from the more rapidly developing aspiration pneumonitis, which usually occurs as a result of chemical injury from aspiration of sterile gastric contents.

There is an argument that most pneumonia involves aspiration of pathogenic organisms, such as Streptococcus pneumoniae and Haemophilus influenzae, which colonise the oropharynx along with anaerobic bacteria. Despite microaspiration of oropharyngeal secretions in community-acquired pneumonia, most patients improve without specific anti-anaerobic antibiotics such as metronidazole. ${ }^{4,6}$

\section{What is the evidence for anaerobic bacteria causing aspiration pneumonia?}

Isolation of anaerobic organisms is difficult, requiring specific sampling, transport and culturing measures. ${ }^{7}$ Anaerobic sensitivity testing is even more difficult to perform and is rarely undertaken on a routine basis. Although recent studies have reported on the microbiology of aspiration pneumonia, they did not always include specific anaerobic organism isolation techniques. ${ }^{8,9}$

The use of metronidazole in combination with penicillin in treating aspiration pneumonia is largely based on several studies from the 1970s that reported predominantly anaerobic flora from percutaneous, transtracheal and thoracocentesis samples in patients with the disease. ${ }^{10-13}$ However, subsequent reviews noted that many of these specimens were obtained after complications such as abscesses, necrotising pneumonia or empyema had

\section{Summary \\ - Aspiration pneumonia occurs most commonly in patients with a predisposition to aspiration (eg, those with neurological bulbar dysfunction). \\ - There is limited evidence regarding the involvement of anaerobes in most cases of aspiration pneumonia. \\ - Most patients respond to treatment for aspiration pneumonia without specific anti-anaerobic therapy such as metronidazole. \\ - Metronidazole has adverse side effects, and widespread use where not indicated can promote carriage of multiresistant intestinal flora such as vancomycin- resistant enterococci. \\ - Use of metronidazole may be appropriate in patients with aspiration pneumonia and evidence of a lung abscess, necrotising pneumonia, putrid sputum or severe periodontal disease.}

developed. ${ }^{6,7,14}$ Many patients in the studies had chronic alcoholism, and most reported having putrid sputum before developing pneumonia. In addition, most of the patients responded clinically to penicillin $G$, despite the presence of penicillin-resistant organisms in the samples obtained, suggesting that not all the cultured bacteria were pathogenic.

Studies in the 1990s used bronchoscopic and protected specimen brush techniques to obtain samples from patients with aspiration pneumonia, but failed to isolate any pathogenic anaerobes, despite reasonable anaerobic culture procedures. The most frequently isolated bacteria from patients with community-acquired aspiration pneumonia were S. pneumoniae, H. influenzae,

Staphylococcus aureus and Enterobacteriacae, while gramnegative flora were predominantly isolated in hospitalacquired cases. ${ }^{7,15}$

Jason C Kwong MB BS, BMedSci, Infectious Diseases Registrar

Benjamin P Howden MB BS, PhD, FRACP, Infectious Diseases Physician and Medical Microbiologist

Patrick G P Charles MB BS, PhD, FRACP, Infectious Diseases Physician

Austin Health, Melbourne, VIC.

jason.kwong@ austin.org.au

MJA 2011; 195: 380-381 doi: 10.5694/mjall.10298
A study in 2003 reported on the microbiology of severe aspiration pneumonia in 95 residents of an aged care facility. ${ }^{14}$ Patients with immunosuppression or who had received recent antimicrobial therapy were excluded. Rigorous sampling was undertaken to identify pathogens, including blood cultures, urinary antigen tests and bronchoscopic methods, with appropriate specimen processing for anaerobic organism detection. Isolates from 11 patients contained anaerobes: eight were penicillinsusceptible (Prevotella, Fusobacterium and Peptostreptococcus) and three penicillin-resistant (Prevotella and Bacteroides). Four of the 11 patients with anaerobic isolates received initial empirical treatment that included specific anti-anaerobic therapy such as metronidazole, while seven patients received empirical treatment deemed inadequate for anaerobic pathogens (although one received ampicillin-sulbactam). Despite this, six of these seven showed clinical improvement. 
What are the current guidelines for treating aspiration pneumonia?

The most recent edition of Therapeutic guidelines: antibiotic (published in 2010) suggests the empirical use of benzylpenicillin in combination with metronidazole for treating patients with aspiration pneumonia. ${ }^{2}$ Similarly, the British Thoracic Society guidelines suggest the use of intravenous amoxicillin-clavulanic acid. ${ }^{3}$ However, the Infectious Diseases Society of America-American Thoracic Society guidelines for treatment of all communityacquired pneumonia state that the need for specific anaerobic coverage is generally overestimated and it is "clearly indicated only in the classic aspiration pleuropulmonary syndrome" in patients with underlying risk factors for aspiration. ${ }^{4}$

To our knowledge, there have been no prospective randomised controlled trials for the treatment of aspiration pneumonia with and without metronidazole.

\section{What is the problem with giving unnecessary metronidazole?}

Aside from the potential side effects of metronidazole, such as abdominal pain, pancreatitis or, more rarely, neurotoxicity, ${ }^{16}$ indiscriminate use may promote antibiotic-resistant flora. As most bacteria present in the bowel are anaerobic, the empirical use of specific antianaerobic therapy such as metronidazole has the potential to cause significant collateral damage. Animal studies have alluded to an association between anti-anaerobic therapy and the development and persistence of vancomycinresistant enterococci (VRE) carriage. ${ }^{17-19}$

The use of specific anti-anaerobic therapy in humans also appears to be linked to higher rates of VRE and resistant gram-negative bacteria. ${ }^{20,21}$ These studies have suggested that commensal anaerobic intestinal flora may inhibit growth of some pathogens, and that the use of antimicrobial agents with broad anti-anaerobic activity facilitates high-density colonisation with resistant pathogens such as VRE, with the potential for easier transmission between patients. ${ }^{22}$ This hypothesis was supported by an Australian study of VRE colonisation, which identified a significant association between the use of antibiotics with anti-anaerobic activity and new detection of VRE. ${ }^{23}$

\section{Recommendations}

The evidence for empirical use of metronidazole appears questionable, given recent data showing that anaerobic organisms may not be as pathogenically significant in most aspiration pneumonia as once thought.

Although metronidazole may not be necessary in initial empirical therapy of aspiration pneumonia, there is a need for appropriate treatment where anaerobic bacteria such as Bacteroides are the primary pathogens. This may manifest with the development of abscesses, empyema or necrotising pneumonia, and treatment of these episodes should include specific anti-anaerobic therapy. Although there is less evidence, patients with putrid sputum or severe periodontal disease who develop aspiration

pneumonia may also benefit from treatment with metronidazole in addition to a $\beta$-lactam antibiotic. ${ }^{6}$

The condition of most patients with pneumonia improves without specific anti-anaerobic therapy such as metronidazole, and given that most pneumonia involves some degree of aspiration, there is an argument for treating aspiration pneumonia similarly to standard community- or health care-acquired pneumonia.

Omission of metronidazole from empirical treatment for most patients with aspiration pneumonia may be of benefit in this era of increasing antibiotic resistance.

Acknowledgements: We thank Peter Ward and Janet Montgomery from the Department of Microbiology, Austin Health, for their advice.

Competing interests: No relevant disclosures.

Provenance: Not commissioned; externally peer reviewed.

1 Johnson PDR, Irving LB, Turnidge JD. Practice essentials - infectious diseases. 3: Community-acquired pneumonia. Med J Aust 2002; 176: 341-347.

2 Antibiotic Expert Group. Therapeutic guidelines: antibiotic. Version 14. Melboume: Therapeutic Guidelines Limited, 2010.

the empirical use of specific anti-anaerobic therapy such as metronidazole has the potential to cause significant collateral damage
3 Lim WS, Baudouin SV, George RC, et al. BTS guidelines for the management of community acquired pneumonia in adults: update 2009. Thorax 2009; 64 Supp 3: $1-55$.

4 Mandell LA, Wunderink RG, Anzueto A, et al. Infectious Diseases Society of America/ American Thoracic Society consensus guidelines on the management of community-acquired pneumonia in adults. Clin Infect Dis 2007; 44 Suppl 2: S27$\mathrm{S} 72$.

5 Teramoto S, Fukuchi Y, Sasaki H, et al. High incidence of aspiration pneumonia in community-and hospital-acquired pneumonia in hospitalized patients: a multicenter, prospective study in Japan. J Am Geriatr Soc 2008; 56: 577-579.

6 Marik PE. Aspiration pneumonitis and aspiration pneumonia. N Engl J Med 2001; 344: 665-67.

7 Mier L, Dreyfuss D, Darchy B, et al. Is penicillin G an adequate initial treatment for aspiration pneumonia? A prospective evaluation using a pro-tected specimen brush and quantitative cultures. Intensive Care Med 1993; 19: 279-284.

8 Ebihara S, Ebihara T, Yamanda S, Kohzuki M. Bacteriology of aspiration pneumonia due to delayed triggering of the swallowing reflex in elderly patients [poster abstract]. Am J Respir Crit Care Med 2010; 181: A6176.

9 Lanspa MJ, LiY, Brown S, et al. Examining infectious pathogens in health-care associated aspiration pneumonia and community acquired aspiration pneumonia [poster abstract].Am J Respir Crit Care Med 2011; 183: A4858.

10 Bartlett JG, Gorbach SL, Finegold SM. The bacteriology of aspiration pneumonia. Am J Med 1974; 56: 202-207.

11 Lorber B, Swenson RM. Bacteriology of aspiration pneumonia: a prospective study of community- and hospital-acquired cases. Ann Intern Med 1974; 81: 329-331.

12 Cesar L, GonzalezCCL,CaliaFM. Bacteriologic flora of aspiration-induced pulmonary infections. Arch Intern Med 1975; 135: 711-714.

13 Bartlett JG, Gorbach SL. Treatment of aspiration pneumonia and primary lung abscess: penicillin G vs clindamycin. JAMA 1975; 234: 935-937.

14 El-Solh AA, PietrantoniC, Bhat A, et al. Microbiology of severeaspiration pneumonia in institutionalized elderly. Am J Respir Crit Care Med 2003; 167: 1650-1654.

15 Marik PE, Careau P. The role of anaerobes in patients with ventilator-associated pneumonia and aspiration pneumonia: a prospective study. Chest 1999; 115: 178183.

16 Chua K, Howden BP. Metronidazole. In: Grayson ML, Crowe SM, McCarthy JS, et al, editors. Kucers' the use of antibiotics. 6thed. London: ASM Press, 2010: 1211-1237.

17 Donskey CJ, Hanrahan JA, Hutton RA, Rice LB. Effect of parenteral antibiotic administration on the establishment of colonization with vancomycin-resistant Enterococcus faecium in the mouse gastrointestinal tract. J Infect Dis 2000; 181: $1830-1833$.

18 Donskey CJ, Hanrahan JA, Hutton RA, Rice LB. Effect of parenteral antibiotic administration on persistence of vancomycin-resistant Enterococcus faecium in the mouse gastrointestinal tract. J Infect Dis 1999; 180: 384-390.

19 Launay A, Ballard SA, Johnson PDR, et al. Transfer of vancomycin resistance transposon Tn1549 from Clostridium symbiosum to Enterococcus spp. in the gut of gnotobiotic mice. Antimicrob Agents Chemother 2006; 50: 1054-1062.

20 Edmond MB, Ober JF, Weinbaum DL, et al. Vancomycin-resistant Enterococcus faecium bacteremia: risk factors for infection. Clin Infect Dis 1995; 20: 1126-1133.

21 Bhalla A, Pultz NJ, Ray AJ, et al. Antianaerobic antibiotic therapy promotes overgrowth of antibiotic-resistant, gram-negative bacilli and vancomycin-resistant enterococci in the stool of colonized patients. Infect Control Hosp Epidemiol 2003; 24: 644-649.

22 Donskey CJ, Chowdhry TK, Hecker MT, et al. Effect of antibiotic therapy on the density of vancomycin-resistant enterococci in the stool of colonized patients. NEngl J Med 2000; 343: 1925-1932.

23 Padiglione AA, Wolfe R, Grabsch EA, et al. Risk factors for new detection of vancomycin-resistant enterococci in acute-care hospitals that employ strict infection control procedures. Antimicrob Agents Chemother 2003; 47: 2492-2498. 